\title{
ZIKA VIRUS: REVIEW OF CURRENT STATUS
}

\author{
KOPPALA RVS CHAITANYA ${ }^{1}$, VENNA R SURYA ANUSHA ${ }^{2 *}$
}

${ }^{1}$ Department of Pharmacology, School of Pharmacy, Guru Nanak Institutions, Ibrahimpatnam -501 506, Telangana, India. ${ }^{2}$ Department of Pharmaceutics, School of Pharmaceutical Sciences and Technologies, Jawaharlal Nehru Technological University, Kakinada - 533 003, Andhra Pradesh, India. Email: rajeswarianusha@gmail.com

Received: 20 February 2018, Revised and Accepted: 10 April 2018

\begin{abstract}
Zika virus (ZIKV) is a mosquito-borne virus which startled the world with its tremendous outbreaks in Africa, America, Asia, and Pacific region. Although the symptoms are mild such as fever and headache, its complications are severe in newborn: Guillain-Barre Syndrome and congenital Zika syndrome including microcephaly. Its ability to transmit through vector and non-vector means especially vertical transmission made it a potential threat. The World Health Organization with the support of other organizations implemented several programs to eradicate the spread of the virus. Development of a vaccine for ZIKV is still under clinical trials. An anti-hepatitis $C$ drug was repurposed for treating infected persons especially pregnant women to limit vertical transmission of ZIKV. This review focuses on the historical background, structure, phylogeny, transmission, symptoms, diagnosis, prevention, and treatment of ZIKV along with strategies planned for control and monitoring of ZIKV.
\end{abstract}

Keywords: Zika virus, Structure, Phylogeny, Guillain-Barre syndrome, Congenital Zika syndrome, Microcephaly, Zika strategic response plan, Zika vaccine, Sofosbuvir, India.

(C) 2018 The Authors. Published by Innovare Academic Sciences Pvt Ltd. This is an open access article under the CC BY license (http://creativecommons. org/licenses/by/4. 0/) DOI: http://dx.doi.org/10.22159/ajpcr.2018.v11i7.25377

\section{BACKGROUND}

Zika virus (ZIKV) is a 70 years back begun story to which still scientists are searching for an end. It was identified in Uganda and stretched over Africa, America, Asia, and the Pacific. It emerged as primary health concern infecting millions of people manifesting neurological complications and congenital malformations. This piece of writing provides an insight into the origin, transmittance, symptoms, complications of Zika viral infection, and treatment and strategies to fight back.

\section{CHRONICLE OF ZIKV}

Virus is named after Zika forest of Uganda in which it was first isolated from a rhesus monkey in 1947 during a study on yellow fever by Dick et al. [1]. In the same study after a series of examinations on mosquito species; virus is documented in Aedes africanus species [1]. Possibility of human infection was assumed by the presence of antibodies in a serological study on natives of Tanzania and Uganda in 1952 [2]. Symptoms of fever and headache have been observed through human subjects in 1954 which provides direct evidence for ZIKV infection [3]. During 1960-1980, no deaths or hospitalizations are reported, but studies showed widespread of Zika infection in humans. The first outbreak of Zika infection in humans occurred in 2007 in Pacific island of Yap, Federated States of Micronesia. A similarity in symptoms of Zika, chikungunya and dengue infections might be a reason for underreporting of Zika outbreaks earlier [4]. A scientist working on mosquito sampling project in Senegal reported being bitten by Aedes spp. mosquitoes every day. After his return to hometown he transmitted the infection to his wife through sexual contact. Unlike the other arboviruses, ZIKV exhibits sexual transmission [5]. During ZIKV outbreak in French Polynesia and south Pacific, newborn reported transplacental transmission [6]. Positive result for ZIKV in asymptomatic blood donors alerted the authorities for establishing ZIKV infection detection and clinical management [7]. Brazil reported neurological disorders in 49 cases associated with Zika infection which was confirmed as Guillain-Barre syndrome. With continues increase in Zika associated microcephaly cases and neurological disorders, the World Health Organization (WHO) declared a national public health emergency in
February 2016 [4]. ZIKV was added to the list of nationally notifiable conditions as subtype of arbovirus in the U.S in January 2016 [8]. The WHO released a document on prevention of sexual transmission of ZIKV on September 2016 which is intended to guide appropriate sexual practices in the context of ZIKV [9]. On March 2016, Zika emergency committee released level 2 warning for pregnant women not to travel to areas with Zika outbreaks along with some recommendations $[4,10]$. As of June 27, 2016, autochthonous transmission of ZIKV has been confirmed in 40 countries and territories within the Americas [11]. To control the distribution of this dangerous virus to non-endemic areas (areas without immunity) WHO designed several strategies for vector control, prevention, and management of medical complications which will be specified in further discussion.

\section{STRUCTURE AND PHYLOGENY}

ZIKV is a member of Flaviviridae family transmitted by mosquitoes so alternatively referred as flavivirus and arbovirus. It shares the same family of human pathogens such as dengue virus, West Nile virus, and yellow fever virus. As all flaviviruses, ZIKV consists of a membrane surrounding the capsid with viral ribonucleic acid (RNA) genome. About 180 copies of both envelope (E) glycoprotein and the membrane (M) protein inserted into lipid membrane constitute the icosahedral capsid. Major viral proteins of ZIKV are structurally and functionally similar to other flaviviruses except at asn 154 glycosylation site which may serve as an attachment point to host cells. From structural elucidation, researches suggested E glycoprotein as a possible target of drugs for therapy $[12,13]$. Although phylogenetic studies revealed a common lineage of American and Asian strains, researches identified a set of amino acid residues that are uncommon between the strains and also from ancient African strains. This might be due to recombination which provided an increase in pathogenicity and transmission efficiency of ZIKV [13]

\section{TRANSMISSION}

Vector transmission

As specified earlier during a study by Dick et al., several varieties of mosquitoes, namely, A. africanus, Aedes furcifer, A. furcifer, Aedes 
luteocephalus, Aedes vittatus, Aedes dalzieli, Aedes hirsutus, Aedes metalicus, Aedes taylori, Aedes aegypti, Aedes unilineatus, Anopheles coustani, Culex perfuscus, Mansonia uniformis, and Taeniorhynchus species, Eretmapodite species of Zika forest were monitored for the presence of virus. Serum suspension prepared from a lot of $A$. africanus caused Zika viral infection in mice, validating the presence of virus in A. africanus species. Yet, it lacks substantial proof as a vector in transmission of ZIKV [1]. Later, Boorman and Porterfield revealed transmission of ZIKV to mice and monkey by A. aegypti [14]. In Asia, further evidence incriminated $A$. aegypti as the urban vector after ZIKV identification in a mosquito pool collected in Malaysia [15]. Furthermore, in Indonesia, the peak in human ZIKV infections coincided with a peak in the A. aegypti population [16]. Vector born transmission is found to include two distinct transmission cycles (a) sylvatic cycle - involving ZIKV transmission between non-human primates and arboreal mosquitoes of forests (b) urban cycle - involving ZIKV transmission between humans and urban mosquitoes. Although A. aegypti and Aedes albopictus are earlier assumed to be the primary vectors involved in urban cycle, later evidence supported $A$. aegypti due to its high vectorial capacity than other species. A study by centers for disease control and prevention establishes that $A$. aegypti reaches their maximum number in June-October each year [17].

\section{Non-vector transmission}

\section{Blood transfusion}

As reported in history during outbreak in French Polynesia about 2.8\% of blood samples from asymptomatic donors contained ZIKV RNA, indicating the chance of transmission by blood transfusion [7]. Similar situation aroused in Puerto Rico where 1\% of asymptomatic donors reported positive for ZIKV in 2016 [18], and Brazil [19] confirming the transmission of ZIKV through blood transfusion.

During the ZIKV infection, the pre-symptomatic period can past from 3 to 12 days. In which viremia can occur and could produce up to 8.1 million copies of virus per milliliter in serum. This is reported to be for longer period in whole blood than in serum. Hence, Food and Drug Administration (FDA) has recognized ZIKV as a transfusion-transmitted infection as it is life-threatening and can incur birth defects and can be transmissible by blood or blood components [8].

\section{Sexual transmission}

ZIKV transmission through sex has been reported in all cases including male to male, female to female, and female to male $[5,20,21]$. Although the duration of transmissibility is unknown, ZIKV RNA has been noticed for 6 months in semen and up to 11 days in vaginal fluids of infected persons [22].

\section{Vertical transmission}

On reported detection of microcephaly in neonates born to symptomatic or asymptomatic ZIKV-infected mothers, researches concentrated on vertical transmission of ZIKV. ZIKV RNA and antigens are identified in amniotic fluid, placenta, and fetal nervous tissue [6]. High quantities of ZIKV are detected in breast milk of infected women arousing a doubt of possible transmission to the baby. Serum sample analysis of infant after breastfeeding through reverse transcriptase-polymerase chain reaction (RT-PCR) technique revealed positive result, but viral replication was not detected on breast milk inoculation ruling out the possibility of transmission through milk. While in case of other flaviviruses as dengue virus and West Nile virus, breast milk transmission is previously reported $[23,24]$.

\section{SIGNS AND SYMPTOMS}

Symptoms of ZIKV often coincides with others arbovirus infections especially dengue virus. Often the symptoms of ZIKV are left unnoticed due to their mildness. Symptoms start with mild headache followed by maculopapular rash on face, neck, upper arms and palms, fever, conductivities, joint pain, and malaise. These symptoms typically last for 7 days [25].

\section{COMPLICATIONS}

Although the symptoms of ZIKV appears to be mild and less attention needed, often ZIKV infection leads to severe complications which could be life-threatening.

\section{Guillain-Barre syndrome}

It can be categorized as an autoimmune disorder as in this body's immune system attacks part of the peripheral nervous system and damages the myelin sheath. This syndrome can affect nerves allied to muscle movement, pain, temperature, and touch. It is a rare condition commonly seen in males of all ages. Symptoms comprise tingling sensation and weakness initiating in legs and spread to arms and face leading to paralysis in some cases. About $20-30 \%$ of patients with affected chest muscles showed difficulty in breathing which can be lifethreatening. $3-5 \%$ of patients die due to paralysis of muscles concerned with important systems. In many of the situations, patients showed complete recovery, even though weakness remains as a part of them. Guillain-Barre syndrome is generally headed by a bacterial or viral infection, vaccine administration, or surgery [26]. This statement can be correlated to the increased cases of Guillain-Barre syndrome during the outbreak of ZIKV in Brazil and other countries. ZIKV infection served as triggering agent for Guillain-Barre syndrome. Diagnosis is through monitoring symptoms and neurological testing including decreased or loss of deep-tendon reflexes. There is no known cure, but treatment can recover symptoms and cut down its duration [26].

Different types of Guillain-Barre syndrome attribute different types of immunity attacks. Main types include acute inflammatory demyelinating polyradiculoneuropathy (AIDP), Miller Fisher syndrome, acute motor axonal neuropathy, and acute motor - sensory axonal neuropathy. In AIDP variant the demyelination is done by white blood cells (T lymphocytes and macrophages) while in case of axonal variants it is mediated by IgG antibodies and compliment against the cell membrane covering axon [27].

As a part of neuroviruses emerging in the Americas study, all the patients diagnosed with Guillain-Barre syndrome from January to March 2016 underwent clinical and neurological evaluation. This study suggested that temporal profile of neurological symptoms related to ZIKV caused Guillain-Barre syndrome is not the same as a syndrome associated with other infections. The syndrome is observed to be parainfectious rather than postinfectious which can be possibly accounted as: ZIKV triggers an immune molecular mimicry against nervous system antigens leading to immune dysfunction and Guillain-Barre syndrome. Furthermore, there is a prolonged period of viruria which even endure after viral syndrome suggesting the use of RT-PCR urine test as a valuable diagnostic tool for ZIKV infection in Guillain-Barre syndrome patients. The study also found a potential relationship between ZIKV associated Guillain-Barre syndrome and previous exposure of the patient to dengue virus. $86 \%$ of patients with Guillain-Barre syndrome had evidence of antibodies against dengue virus indicating prior exposure to dengue virus, and ZIKV has been a secondary flavivirus infection. Most of the patients in the study had AIDP form of Guillain-Barre syndrome the reason for which is yet to be revealed [28].

\section{Microcephaly}

Microcephaly is a birth defect where the size of baby's head is smaller than anticipated. In gestation period, the head size depends on brain growth. Microcephaly occurs when baby's brain has not developed during pregnancy, or brain growth stopped due to injury during pregnancy. Depending on severity microcephaly is coupled with other problems such as seizures, developmental delay, intellectual disability, difficulty in swallowing, imbalance, hearing loss, and vision problems. Severe microcephaly can be life-threatening and often needs close monitoring by regular check-ups [29].

ZIKV increased the incidence of microcephaly in babies born to infected mothers in various regions which called the need for a study on microcephaly in ZIKV-infected pregnant women. Mlakar et al. through his study reported intrauterine transmission of virus causing severely 
affected central nervous system (CNS) and growth retardation. Virus damaged the placenta which was confirmed through calcification of placenta and low placental-fetal weight ratio. ZIKV is primarily found to be neurotropic in nature, which is also reported in other flaviviruses. Remains of replication complexes are diagnosed in the damaged endoplasmic reticulum of nerve tissues indicating fetal brain as the site of replication of ZIKV. The viral copies are several times higher in fetal brain than reported in adult infected serum but similar to the infected semen samples [30].

In 2017 research revealed the underlying relationship between the ZIKV infection and microcephaly in newborns. Cerebral cortex formation involves neuron formation through direct and indirect methods. In direct method, neurons born from the symmetric division of apical progenitors (APs), while in indirect method APs convert to intermediate progenitors (IPs) which divide to form neurons. A balance exists between direct and indirect neurogenesis regulated by unfolded protein response (UPR). Disruption of this balance leads to microcephaly in humans. ZIKV is vertically transmitted to fetal cortical region targeting APs, but it did not induce apoptosis instead it interrupted the conversion of APs to IPs, hence leading to decreased neuronal output in the infected developing cortex. Any interference with codon translation induced endoplasmic reticulum stress and the UPR which the reason for the hindrance of APs conversion to IPs. UPR is mediated by three pathways, inhibition of these pathways could prevent the chance of microcephaly in newborns of infected women by inhibiting the UPR induced apoptosis [31].

\section{Congenital ZIKA syndrome}

Congenital Zika syndrome includes all birth defects caused by Zika viral infection before birth and is mainly featured by severe microcephaly (earlier discussed), reduced brain tissue including subcortical calcifications, macular scarring and focal pigmentary retinal mottling, arthrogryposis multiplex congenital (curving of joints), and hypertonia (restricted body movements due to CNS damage). The syndrome is also associated with hydrocephalus, redundant scalp skin, tremors, seizures, and irritability [32]. Ophthalmological studies on neonates born with ZIKV infection revealed severe eye abnormalities such as optic nerve hypoplasia, congenital glaucoma, microphthalmia, cataracts, lens subluxation, and intraocular calcifications [33].

\section{DIAGNOSIS}

Asymptomatic nature of ZIKV-infected subjects and its potential to transmit through blood transfusion, sexual contact, and congenital transmission elicited the urgency for the development of the diagnostic method. As no licensed laboratory test for biological quantification of ZIKV is available but as a necessity to control the outbreak is present, FDA has issued emergency use authorization for several diagnostic tools on February 26, 2016, such as Trioplex real-time RT-PCR assay, Zika-MAC ELISA, and real-time Zika test [34].

Multiple assays are to be performed to diagnose the ZIKV infection due to temporal nature of virus in an infected person. Viral RNA can be detected in infected person in earlier days of infection followed by rise in immunoglobulin M (IgM) levels in the blood with a decline in viral RNA. Conversely, viral RNA may be detectable for longer period in some specimens. Nucleic acid testing (NAT) is most relying in first 6 weeks after onset of symptoms while IgM antibodies can be quantified in first 12 weeks and may stick longer. Consequently, diagnostic tests are developed for identification of ZIKV RNA and IgM antibodies in samples. Serum and urine are authorized diagnostic specimens pursued by plasma, whole blood, cerebrospinal fluid (CSF), and amniotic fluid.

Chance for coinfection of ZIKV with other related flavivirus is possible though rare, so testing of other viruses should be considered. Preconception screening is not mandatory for ZIKV unless pregnant women were either symptomatic or asymptomatic with a history of exposure or ongoing exposure of virus. For symptomatic pregnant women with possible previous exposure to ZIKV, NAT along with IgM serological test is preferred. In case of asymptomatic pregnant women with ongoing exposure, NAT is suggested for 3 times [35].

Samples with negative NAT result for ZIKV and nonnegative or positive result for the serological test are forwarded to Center for Disease Control and Prevention to confirm the infection through plaque reduction neutralization test (PRNT). PRNT is based on the serum concentration needed to reduce the plaques by $50 \%$ compared to virus-free serum. It gives a measure of antibodies present and effectiveness of antibodies in terms of PRNT $_{50}$ value [35]

\section{TREATMENT}

As no specific treatment is available for ZIKV infection, people are advised to rest and drink enough fluids. Symptoms such as fever and pains are treated with common medicines. Research is being done for the development of vaccines and medicines [4]

\section{PREVENTION}

As a response to the severe outbreak of ZIKV and congenital abnormalities of newborns, the WHO has released some recommendations for travelers and pregnant women for emergency control of ZIKV transmission [36].

- Mosquitoes being the vector of transmission, measure to be taken for their population control using insect repellents containing diethyltoluamide, KBR3023 (Icaridin or Picaridin), and IR 3535 ((3-[N-butyl-N-acetyl] and aminopropionic ethyl-ester).

- During travel to infected areas, wear clothes that cover maximum body parts and use mosquito nets while sleeping especially during daytime (as Aedes species is active during this time).

- All travelers returning from infected areas are advised to practice safe sex using condoms or avoid sex for 6 months, as some of them can be asymptomatic. They are also advised to use insect repellents for 3 weeks to avoid the chance of spreading the infection to other persons.

- Pregnant women (in infected areas or with partner traveling back from infected areas) should practice safer sex or should stay away from sex during the duration of pregnancy [36].

On 14-15 March 2016, the WHO assembles vector advisory group to design tools for effective control of ZIKV transmission through Aedes mosquitoes. Two new tools were referred for pilot development by them: Wolbachia-infected A. aegypti and Oxitec transgenic mosquitoes. Wolbachia is a bacterium that stops chikungunya, dengue, and ZIKV replication in mosquitoes. This is naturally present in $60 \%$ of insect species but not in Aedes. Wolbachia-infected mosquitoes when mate with normal mosquitoes, they pass the bacterium to next-generation forbidding vector for the virus. Scientists released large quantities of Wolbachia-infected mosquitoes in Rio during Olympics 2014. Oxitec transgenic mosquitoes are genetically modified mosquitoes that have a gene which prevents their offspring from existing up to maturity. Only male mosquitoes are released so that there will not be any possibility of transmission of disease, as females only bite humans. On mating with wild females, they pass this gene to further generations [37].

\section{ZIKA STRATEGIC RESPONSE PLAN}

WHO and Pan American Health Organization started working together on ZIKV long before Zika outbreak. On February 2016, they launched global strategic response plan for appropriate action. The plan was revised on June 7, 2016, for a period July 2016-December 2017. The plan aims to support national governments and communities for preventing and controlling medical complications caused by ZIKV infection. About 23 partners joined the WHO to implement the plan which later increased to 60 . The agency has triggered an incident management system in its headquarters, Geneva and regional offices to implement its health emergencies program. Government of affected areas advised women to postpone conceiving and to avoid traveling. Zika response portal is established on the WHO website which served as a central point of reference for all partners. It ensures the direction 
of help to the needed without duplication and deficits. Immediate priority of funding is given to preventing and managing the medical complications, expanding health system's competence, sexual and reproductive health risk and integrated vector management.

To achieve the objectives five strategies are employed: Detection, prevention, care and support, research, and coordination. Detection concentrates on integrated surveillance systems to provide up to date information, to guide response. Prevention - prevent adverse outcomes of ZIKV by integrated vector management, risk communication, and community engagement. Care and support involve strengthening of health and social systems at different levels to offer services and support to affected. Research includes interventions to prevent, detect, and control ZIKV infection and manage its complications. It also encloses research on Aedes mosquito control tools, vaccines, and diagnostic tests. Coordination involves establishment and maintenance of adequate, transparent coordination for accurate response to Zlka infection [10].

First quarterly report on Zika strategic response plan was released enclosing the latest information on different strategies of the plan. These updates are for guidance, coordination, and collaboration among the WHO and its partners to support countries [38].

\section{VACCINE DEVELOPMENT}

Vaccination is the ideal remedy for ZIKV infection. WHO along with UNICEF developed ZIKV vaccine target product profile for use in an emergency scenario in July 2016. About 30 organizations, including 8 public sector institutions are engaged in vaccine development. Two DNA vaccines (by GeneOne Life Sciences Inc and National Institute of Allergy and Infectious Diseases [NIAID] in the United States) reached Phase I trials while many other candidates are about to move to Phase I trials $[38,39]$.

Synthetic DNA vaccine that targets the ZIKV premembrane and envelope proteins are prepared by GeneOne life sciences and are delivered through CELLECTRA-3P electroporation device. The vaccine reached Phase I on producing cellular and humoral immune responses along with the production of neutralizing antibodies, in mice and nonhuman primates. In the Phase I, open-label clinical trial, it elicited anti-ZIKV immune responses, achieving a clearance for Phase I. However, studies on further safety and efficacy are yet to be performed [40].

\section{THERAPY BY REPURPOSING APPROVED DRUG}

While the world is busy in designing immunization strategies through vaccine development, some researches concentrated on the screening of FDA approved drugs to repurpose for ZIKV. Need to cure infected persons and avoid vertical transmission in already infected pregnant women (in whom prevention is no longer an option) insisted the search for a drug to cure Zika infection. Sofosbuvir (SOF), a RNA dependent RNA polymerase inhibitor was a FDA approved a drug to treat and cure hepatitis C infections. SOF intracellularly converts into its triphosphate and inhibits RNA polymerase of hepatitis C virus (HCV). RNA polymerase of ZIKV and HCV is similar in their active sites. Due to the phylogenic and structural similarity of HCV and ZIKV, SOF are tested for possible action on ZIKV. SOF tested on infected neural progenitor cells, and three-dimensional neurospheres prevented cell death by ZIKV infection. In vivo testing on immunodeficient mouse models also showed promising results. Efficacy of SOF in blocking vertical transmission in ZIKV permissive SJL strain21 was tested. Decrease in serum concentration of ZIKV was reported in pregnant SJL strain21 along with blocking of vertical transmission [41].

\section{ZIKA IN INDIA}

On September 30, 2017, Indian Council of Medical Research (ICMR) has released ZIKV update revealing the preparedness and response. ICMR has conducted three training programs for 25 laboratories to diagnose ZIKV. Human surveillance network for ZIKV was established to screen the human serum/blood and urine samples of suspected persons, patients with dengue and chikungunya and pregnant women. About 45,820 samples are tested through this surveillance till the date of which three different individuals found to be positive for ZIKV. (1) 34-yearold female developed low-grade fever 1 week after delivering healthy baby and tested negative for dengue, chikungunya fever but positive for ZIKV. (2) 22-year-old pregnant female in 37 weeks of pregnancy was tested positive and (3) 64-year-old male with febrile illness of 8 days duration tested negative for dengue fever and positive for ZIKV. (4) 27-year-old male with acute febrile illness for 3 days was tested positive for ZIKV in urine, but blood was negative. Further confirmation by PCR showed 98\% sequence similarity with ZIKV Asian lineage. Since July 2016, three ICMR institutes initiated vector surveillance in different parts of India and tested 25,960 pools of mosquitoes by RTPCR technique, but no sign of ZIKV was reported.

The reproductive and child health division of ministry of health and family Welfare has initiated programs on newborn birth defect screening and stillbirth surveillance, under Rashtriya Bal Swasthya Karyakram. It focuses on reporting 8 externally visible birth defects including microcephaly. ICMR and Monash University has signed MoA on February 2017 regarding strategies for vector control in India [42]. Several such programs are being successfully implemented by Indian government agencies in collaboration with the WHO.

\section{CONCLUSION}

Due to high complications and multiple modes of transmission of ZIKV, there is a necessity for improving knowledge on ZIKV among the healthcare professionals and the general public to regulate the spread of infection. Government of all countries along with the WHO and several other organizations are implementing programs to effectively eradicate ZIKV infection. Programs on assessing awareness among people are also necessary for effective functioning of Zika strategies [43]. Approval of vaccine can keep an end to the story of ZIKV.

\section{AUTHOR'S CONTRIBUTIONS}

Authors equally contributed to articles collection and framing of manuscript. Each author has reviewed and revised the article for efficient presentation of viewpoint.

\section{CONFLICTS OF INTEREST}

The authors declared that they have no conflicts of interest.

\section{REFERENCES}

1. Dick GW, Kitchen SF, Haddow AJ. Zika virus. I. Isolations and Serological Specificity. Trans R Soc Trop Med Hyg. 1952;46:509-20.

2. Smithburn KC. Neutralizing antibodies against certain recently isolated viruses in the sera of human beings residing in East Africa. J Immunol 1952;69:223-34.

3. Macnamara FN. Zika virus: A report on three cases of human infection during an epidemic of jaundice in Nigeria. Trans R Soc Trop Med Hyg 1954:48:139-45.

4. Kindhauser MK, Allen T, Frank V, Santhana R, Dye C. Zika: The origin and spread of a mosquito-borne virus. Bull World Health Organ 2016. DOI: org/10.2471/BLT.16.171082

5. Foy BD, Kobylinski KC, Foy JL, Blityich BJ, Rosa AT, Haddow AD, et al. Probable non-vector-borne transmission of zika virus, Colorado, USA. Emerg Infect Dis 2011;17:880-2.

6. Besnard M, Lastère S, Teissier A, Cao-Lormeau VM, Musso D. Evidence of perinatal transmission of Zika virus, French polynesia. Euro Surveill 2014;19:pii20751.

7. Musso D, Nhan T, Robin E, Roche C, Bierlaire D, Zisou K, et al. Potential for zika virus transmission through blood transfusion demonstrated during an outbreak in french polynesia, november 2013 to february 2014. Euro Surveill 2014;19:pii:20761.

8. FDA. Revised Recommendations for Reducing the Risk of Zika Virus Transmission by Blood and Blood Components. Maryland, US. August 2016. Available from: https://www.fda.gov/downloads/ biologicsbloodvaccines/guidancecomplianceregulatoryinformation/ guidances/blood/ucm518213

9. WHO. Prevention of Sexual Transmission of Zika Virus. Interim 
Guidance Update. Geneva. 6 September 2016. WHO/ZIKV/MOC/16.1 Rev. 3. Available from: http:/www.who.int/csr/resources/publications/ Zika/sexual-transmission-prevention/en.

10. WHO. Zika Strategic Response Plan Revised For July 2016-December 2017. Geneva. June 2016. Available from: http://www.apps.who. int/iris/bitstream/10665/246091/1/WHO-ZIKV-SRF-16.3-eng. pdf?ua $=1 \& u a=1 \& u a=1 \& u a=1$

11. Bushak L. A Brief History of Zika Virus, from its Discovery in the Zika Forest to the Global Outbreak Today. Medical Daily. 8 April 2016. Available from: http://www.medicaldaily.com/Zika-virus-outbreakhistory-381132.

12. Sirohi D, Chen Z, Sun L, Klose T, Pierson TC, Rossmann MG, et al. The $3.8 \AA$ resolution cryo-EM structure of zika virus. Science (New York, NY) 2016;352(6284):467-70

13. Ye Q, Liu ZY, Han JF, Jiang T, Li XF, Qin CF. Genomic characterization and phylogenetic analysis of Zika virus circulating in the Americas. Infect Genet Evol 2016;43:43-9.

14. Boorman JP, Porterfield JS. A simple technique for infection of mosquitoes with viruses; transmission of Zika virus. Trans R Soc Trop Med Hyg 1956;50:238-42.

15. Marchette NJ, Garcia R, Rudnick A. Isolation of Zika virus from Aedes aegypti mosquitoes in Malaysia. Am J Trop Med Hyg 1969;18:411-5.

16. Olson JG, Ksiazek TG, Triwibowo S. Zika virus, a cause of fever in central Java, Indonesia. Trans R Soc Trop Med Hyg 1981;75:389-93.

17. Song BH, Lee YM. Zika virus: History, epidemiology, transmission, and clinical presentation. J Neuroimmunol 2017;308:50-64.

18. Kuehnert MJ, Basavaraju SV, Moseley RR, Pate LL, Galel SA, Williamson PC, et al. Screening of blood donations for Zika virus infection-Puerto Rico. Morb Mortal Wkly Rep 2016;24:65:627-8.

19. Barjas-Castro ML, Angerami RN, Cunha MS, Suzuki A, Nogueira JS, Rocco IM, et al. Probable transfusion-transmitted Zika virus in Brazil. Transfusion 2016;56:1684-8.

20. Deckard DT, Chung WM, Brooks JT, Smith JC, Woldai S, Hennessey M, et al. Male-to-male sexual transmission of Zika virus-texas. MMWR Morb Mortal Wkly Rep 2016;65:372-4

21. Davidson A, Slavinski S, Komoto K, Rakeman J, Weiss D. Suspected female-to-male sexual transmission of zika virus-New York City, 2016. MMWR Morb Mortal Wkly Rep 2016;65:716-7.

22. Barzon L, Pacenti M, Franchin E, Lavezzo E, Trevisan M, Sgarabotta D, et al. Infection dynamics in a traveller with persistent shedding of Zika virus RNA in semen for six months after returning from Haiti To Italy, January 2016. Euro Surveill 2016;21:pii30316.

23. Ognjan A, Boulton ML, Somsel P, Stobierski MG, Stoltman G, Downes $\mathrm{K}$, et al. Possible west Nile virus transmission to an infant through breast-feeding-Michigan. MMWR Morb Mortal Wkly Rep 2002;51:877-8

24. Barthel A, Gourinat AC, Cazorla C, Joubert C, Dupont-Rouzeyrol M, Descloux E. Breast milk as a possible route of vertical transmission of dengue virus? Clin Infect Dis 2013;57:415-7.

25. Lanciotti RS, Kosoy OL, Laven JJ, Velez JO, Lambert AJ, Johnson AJ, et al. Genetic and serologic properties of zika virus associated with an epidemic, yap state, Micronesia, 2007. Emerg Infect Dis 2008;14:1232-9.

26. WHO. Guillain-Barré Syndrome, Fact Sheet Geneva. October 2016. Available from: http://www.who.int/mediacentre/factsheets/guillain- barre-syndrome/en/.

27. Van Doorn PA, Ruts L, Jacobs BC. Clinical features, pathogenesis, and treatment of guillain-barré syndrome. Lancet Neurol 2016;7:939-50.

28. Parra B, Lizarazo J, Jiménez-Arango JA, Zea-vera AF, GonzálezManrique G, Vargas J, et al. Guillain-barré syndrome associated with Zika virus infection in Colombia. N Engl J Med 2016;375:1513-23.28.

29. Center for Disease Control and Prevention. Facts about Microcephaly. Georgia, United States. November 2017. Available from:29. https:// www.cdc.gov/ncbddd/birthdefects/microcephaly.html.

30. Mlakar J, Korya M, Tul N, Popoyic M, Prijatelj MP, Mraz J. Zika virus associated with microcephaly. N Engl J Med 2016;374:951-8.

31. Gladwyn-Ng I, Cordón-Barris L, Alfano C, Creppe C, Couderc T, Morelli G. Stress-induced unfolded protein response contributes to Zika virus-associated microcephaly. Nat Neurosci 2018;21:63-71.

32. Center for Disease Control and Prevention. Congenital Zika Syndrome and Other Birth Defects. Georgia, United States. November 2017. Available from: https://www.cdc.gov/pregnancy/Zika/testing-followup/Zika-syndrome-birth-defects.html.32

33. Beckham JD. Zika virus is associated with microcephaly and eye abnormalities in neonates. Neurol Rev 2016;24:13.

34. U.S. Department of Health and Human Services. Food and Drug Administration. Emergency Use Authorizations, Maryland 2016. Available from: https://www.fda.gov/MedicalDevices/Safety/ EmergencySituations/ucm161496.htm\#Zika.34.

35. Centers for Disease Control and Prevention. Guidance for US Laboratories Testing for Zika Virus Infection. Georgia, United States. July 24, 2017. Available from: https://www.cdc.gov/Zika/laboratories/lab-guidance.html.

36. WHO Information for Travellers Visiting Zika Affected Countries. Geneva. April 2017. Available from: http://www.who.int/csr/disease/ Zika/information-for-travelers/en/.

37. WHO. Promising New Tools to fight Aedes mosquitoes. Bull World Health Organ 2016;94:562-3. Available from: http://www.who.int/ bulletin/volumes/94/8/16-020816.pdf.

38. WHO. Zika Strategic Response Plan Quarterly Update. Geneva. October 2016. Available from: http://www.who.int/emergencies/Zikavirus/response/en/.

39. Abdullahi HS, Umar SH. An overview of recombinant vaccine technology, adjuvants and vaccine delivery methods. Int J Pharm Pharm Sci 2016;8:19-24. Available from: https://www.innovareacademics.in/ journals/index.php/ijpps/article/view/14311/7769.39.

40. Tebas P, Roberts CC, Muthumani K, Reuschel EL. Safety and immunogenicity of an anti-zika virus DNA vaccine-preliminary report. N Engl J Med 2017. DOI: 10.1056/NEJMoa1708120.

41. Mesci P, Macia A, Moore SM, Shiryaev SA, Pinto A, Huang CT. Blocking zika virus vertical transmission. Sci Reports 2018;8:1218

42. Indian Council of Medical Research Division of Epidemiology and Communicable Diseases. Zika Virus Preparedness and Response. Delhi; 2017. Available from: http://www.who.int/csr/don/26-may2017-Zika-ind/en/

43. Akunne MO, Chukwueke UC, Anosike C. Assessment of knowledge of zika virus infection among health care professionals in a Southeastren state of Nigeria. Int J Pharm Pharm Sci 2018;10:56-60. Available from: https://www.innovareacademics.in/journals/index.php/ijpps/article/ view/22634/13851. 